\title{
Predictors of Incomplete Flexible Sigmoidoscopy
}

\author{
Kalyanakrishnan Ramakrishnan, MD, and Dewey C. Scheid, MD, MPH
}

Objectives: Flexible sigmoidoscopy (flex sig) is an easily administered method of screening for colorectal polyps and cancer. In some patients, the depth of insertion is incomplete, which may result in missed polyps and cancers. To address the question of prospective patient selection for this procedure, we analyzed the factors affecting depth of insertion of sigmoidoscopies performed in outpatients over a 3 -year period.

Study Design: The study involved retrospective chart review of procedures performed by one endoscopist over a 3-year period.

Outcomes Measured: Variables that might affect the extent of depth of insertion of the flexible sigmoidoscope.

Results: We developed separate logistic regression models of incomplete depth of insertion for women and men because sex was an effect modifier for many factors. For women, incomplete depth of insertion was related to inadequate preparation [odds ratio (OR) 3.59; 95\% confidence interval (CI), 1.66 to 7.78 ]. Comparisons were made with the lowest risk group-women younger than 70 years with no hysterectomy. For women younger than $\mathbf{7 0}$ years, those with a hysterectomy were more likely to have an incomplete examination (OR 6.89; 95\% CI, 2.68 to 17.73). For women 70 years and older, the odds ratio for women with a hysterectomy $(0 \mathrm{R} 2.68$; $95 \% \mathrm{CI}, 0.96$ to 7.46$)$ was similar to that of women without a hysterectomy (OR 4.79; 95\% CI, 2.27 to 10.12). For men, incomplete depth of insertion was related to age older than 75 years (OR 6.51; 95\% CI, 1.72 to 30.40), history of abdominal surgery (OR 3.15 ; 95\% CI, 0.95 to 10.41), and weight loss (OR 9.62; 95\% CI, 1.98 to 46.67).

Conclusions: Our study showed a relationship between incomplete examination and increasing age, female sex (more than $75 \%$ of the incomplete examinations were in women), poor bowel preparation (in women), hysterectomy, abdominal surgery (in men) and weight loss (in men). Further research is necessary to determine whether a predictive model can be developed that would be useful to select patients most appropriate for flex sig. In those patients in whom difficulty is anticipated, the choice can be made in to perform flex sig under sedation, analgesia, with the help of distraction techniques, or offer primary colonoscopy. (J Am Board Fam Pract 2003;16:478-84.)

Most colorectal cancers arise from adenomatous polyps. ${ }^{1}$ Their long asymptomatic phase allows for timely screening and adequate treatment of these premalignant lesions. Although screening colonoscopy is regarded as the ideal mechanism of detecting polyps because of its completeness and therapeutic potential, significant logistic barriers prevent its widespread implementation. Studies evaluating colonoscopy have suggested that screening flexible sigmoidoscopy (flex sig) using the $60-\mathrm{cm}$ instrument has the capability of detecting $65 \%$ to $75 \%$ of polyps and $40 \%$ to $65 \%$ of colorectal cancers. ${ }^{2-4}$

Submitted, revised, 18 March 2003.

From the Department of Family and Preventive Medicine, University of Oklahoma Health Sciences Center. Address correspondence to K. Ramakrishnan, MD, Department of Family and Preventive Medicine, University of Oklahoma Health Sciences Center, 900 N.E. 10th Street, Oklahoma City, OK 73104. (e-mail: kramakrishnan@ ouhsc.edu).
The procedure can be performed by many physicians and physician extenders with relatively less investment in training and equipment. Hence, flex sig remains an important means of screening a major segment of the population at risk for colorectal cancer.

Incomplete examination is an unfortunate drawback of flex sig, because the procedure is routinely offered without sedation or analgesia for simplicity and ease of administration. Olynyk et $\mathrm{al}^{5}$ noted that $30 \%$ of patients had a depth of insertion of less than $50 \mathrm{~cm}$. Stewart et $\mathrm{al}^{6}$ suggest a $25 \%$ incomplete examination rate and technical difficulty in up to one third of the cases. Painter et $\mathrm{al}^{7}$ found that in up to a quarter of the patients, the descending colon was not intubated. Using radiopaque clips, Lehman et $\mathrm{al}^{8}$ noted that a $60-\mathrm{cm}$ examination reached the splenic flexure in only $33 \%$ of patients, a 50 - to $55-\mathrm{cm}$ examination reached the sigmoid/ descending colon junction in most instances, a 40- 
to $45-\mathrm{cm}$ examination the mid to upper sigmoid, and a $30-$ to $35-\mathrm{cm}$ examination the lower to mid sigmoid. Incomplete examination may result from various factors including: patient symptoms (abdominal pain, constipation), low pain threshold, prior abdominal or pelvic surgery, poor bowel preparation, coexisting bowel pathology aggravating pain (diverticula, colitis), or poor endoscopic technique. $^{6-9}$

The implications of incomplete examination are enormous in that this may result in missed polyps and cancers. A colonoscopic survey of colorectal adenomas by Gillespie et $\mathrm{al}^{10}$ showed that $47.5 \%$ of adenomas and polypoid cancers (40\% of adenomas) were in the sigmoid colon, and $21.5 \%$ of the adenomas and polypoid carcinomas $(19.2 \%$ of the adenomas) were situated in the descending colon. Shinya and Wolff $^{11}$ showed that $46.3 \%$ of polyps (27\% tubulovillous and 7\% villous) were in the sigmoid colon and $24.3 \%$ of the polyps $(28.3 \%$ tubulovillous and $9.4 \%$ villous) were in the descending colon. Analysis of a case series of 751 colonoscopies showed that $45 \%$ of the polyps were in the rectum and sigmoid and a further $13 \%$ were in the descending colon. ${ }^{12}$ Thus, more proximal polyps in the sigmoid and descending colon are missed with various degrees of incomplete flexible sigmoidoscopy. Furthermore, Lieberman et al, ${ }^{13}$ in a study among 3121 patients in the Department of Veterans Affairs system, found that $80 \%$ of patients with advanced adenomas in the proximal colon had an index lesion distal to the splenic flexure. However, only two thirds had index lesions below the descending colon, attesting to the fact that missing polyps on the left side as a result of incomplete sigmoidoscopy also leads to undetected proximal pathology.

Therefore, every attempt needs to be made to attain as complete an examination as possible and to identify those patients who would benefit from having flex sig under sedation or analgesia or those who should have an initial screening colonoscopy. To address this question of prospective patient selection, we looked at factors affecting depth of insertion of flex sig performed in outpatients over a 3-year period.

\section{Methods}

Data were collected from patients who underwent flex sig between November 1998 and January 2002.
Ten community physicians (general internists and family physicians) belonging to 5 different practices referred all patients for either screening or diagnostic flex sig. The principal investigator, a boardcertified family physician with extensive prior surgical and endoscopic (esophagogastroduodenoscopy, flexible sigmoidoscopy, and colonoscopy) experience, using either a 60-cm Olympus OES fiberoptic sigmoidoscope or a Pentax fiberoptic sigmoidoscope, performed all procedures. Bowel preparation was with $10 \mathrm{oz}$ of magnesium citrate taken the previous evening. Patients were encouraged to stay on a fluid diet the day of the procedure and to administer 2 phosphate enemas, one 2 hours before the procedure and the other an hour before the procedure. No sedation was given and no specific distraction methods were used to minimize pain.

\section{Data Collection}

A data collection form was administered to the patient by the physician before flex sig. Data extracted for the purposes of the study included: age, sex, weight, comorbid illnesses, history of prior abdominal and pelvic surgeries, family history of colon cancer or polyps, and prior flex sigs or colonoscopies. Questions regarding symptoms during the year before the flex sig included: abdominal pain, rectal bleeding, and abdominal distention, constipation, diarrhea, anorexia, and weight loss (criteria for the presence of symptoms within the last year included new onset of symptoms, change in pattern of bowel movements within the past year, or recurrent symptoms within the past year considered to be a problem by the patient). Note was made of the adequacy of the bowel preparation (ability to visualize $90 \%$ of the surface area of the bowel up to the depth of scope insertion), depth of insertion of the sigmoidoscope, any limitations to adequacy of examination (pain, pathology, preparation), and whether the procedure had been terminated because of patient discomfort, poor bowel preparation, or pathology seen. Any pathology seen was recorded.

\section{Analysis}

Statistical analyses included descriptive analysis (Student's $t$ test for difference of means and $\chi^{2}$ for proportions), univariate relative odds, and multivariate logistic regression. A multivariate logistic regression model was created to simultaneously 
Table 1. Characteristics of Patients Having Flexible Sigmoidoscopy $(\mathrm{n}=511)$

\begin{tabular}{|c|c|c|}
\hline & Number & Percentage \\
\hline \multicolumn{3}{|l|}{ Sex } \\
\hline Male & 240 & 47 \\
\hline Female & 271 & 53 \\
\hline \multicolumn{3}{|l|}{ Indication } \\
\hline Diagnostic & 23 & 4.5 \\
\hline Screening & 488 & 94.5 \\
\hline \multicolumn{3}{|l|}{ Depth of insertion $(\mathrm{cm})$} \\
\hline 0 to 25 & 8 & 1.6 \\
\hline 25 to 49 & 72 & 14.1 \\
\hline$\geq 50$ & 426 & 83.3 \\
\hline Missing & 5 & 0.1 \\
\hline \multicolumn{3}{|l|}{ Problems during examination } \\
\hline None & 363 & 71.3 \\
\hline Pain & 68 & 13.4 \\
\hline Poor bowel preparation & 60 & 11.8 \\
\hline Pathology interfered & 15 & 2.9 \\
\hline \multicolumn{3}{|l|}{ Pathology seen* } \\
\hline Polyps & 44 & 8.6 \\
\hline Diverticula & 139 & 27.2 \\
\hline Colitis & 22 & 3.97 \\
\hline Hemorrhoids & 147 & 26.6 \\
\hline No pathology & 226 & 46.4 \\
\hline
\end{tabular}

* Multiple pathologies were seen in 74 patients.

consider the relationship between incomplete depth of insertion and factors thought to be associated based on univariate analysis. An incomplete examination was defined as the depth of insertion of less than $50 \mathrm{~cm}$. This measure was chosen because most community practitioners and trainees are more likely to achieve this depth of insertion. A backward elimination technique (likelihood-ratio test for variable removal, $P<.1$ ) was used to evaluate the best model. Because sex was an effect modifier for multiple factors, including several third-level interactions, separate models were created for men and women for ease of interpretation. Analysis of residuals did not suggest major analytic limitations because of violations of model assumptions. Statistical analysis was performed using the SPSS 10.0 program (SPSS Inc., Chicago, IL).

\section{Results}

A total of 511 patients underwent flex sig. The mean age was 60 years (SD, 9.7) A few patients $(\mathrm{n}=$ 23) had a diagnostic flex sig for recent rectal bleeding or abdominal pain; the rest were screening flex sigs (Table 1). Most of the patients $(n=266)$ were asymptomatic when presenting for the examination. The most common abdominal symptoms at presentation included constipation in $13.3 \%$ of the patients $(\mathrm{n}=68)$, rectal bleeding in $13.5 \%(\mathrm{n}=$
69), and abdominal pain in $12.9 \%(\mathrm{n}=66)$. Prior abdominal surgery had been performed in $32.6 \%$ of the patients $(n=166)$, and $42.8 \%$ of the women $(\mathrm{n}=114)$ had undergone a hysterectomy. Twelve percent of the patients $(n=62)$ had undergone previous flexible sigmoidoscopy (either screening or diagnostic) and 3.4\% ( $\mathrm{n}=17)$ had undergone previous colonoscopy. Complete depth of insertion (50 $\mathrm{cm}$ or more) was achieved in $83.3 \%(\mathrm{n}=426)$. In an additional $14.1 \%$ patients $(\mathrm{n}=72)$, depth of insertion was between 25 and $49 \mathrm{~cm}$.

Nearly three fourths of patients $(n=363)$ experienced no problems during the procedure. Pain was the most common problem experienced. In 15 patients, the presence of large diverticula, bowel spasm, inability of the bowel to distend, and/or poor rectal tone interfered with the passage of the sigmoidoscope. The bowel preparation was considered adequate in $84.3 \%$ of the patients $(n=429)$. Poor bowel preparation contributed to a difficult examination in $11.8 \%$ of patients $(\mathrm{n}=60)$. Pain was a significant limiting factor for $21.9 \%$ of women $(n=59)$. Most patients $(n=438)$ expressed satisfaction with the procedure. Of the 67 who did have an incomplete examination, 57 were women. In 37 women (58\%), pain was the limiting factor accounting for dissatisfaction with the procedure.

No pathology was observed in half the patients. Hemorrhoids (26.6\%) and sigmoid diverticula (27.2\%) were the most common abnormalities found. Nearly $10 \%$ of the patients $(n=44)$ were found to have polyps. Most pathology was seen in patients who were asymptomatic at presentation $(\mathrm{n}=144)$. Men had more than twice the number of polyps as women $[12.9 \%$ vs $4.8 \%(P=.001)]$.

The results of univariate analysis of characteristics of patients according to the adequacy of depth of sigmoidoscope insertion is shown in Table 2 . The flex sig was incomplete in $15.8 \%$ of patients $(n=80)$. More than three quarters of the incomplete sigmoidoscopies were in women $(P<.001)$. Patients with incomplete exams were older (64.1 vs 59.5 years; $P<.001)$ and weighed less $(176.0$ vs 188.8 lbs.; $P=.021)$. Patients with incomplete examinations had a greater proportion of all symptoms, especially abdominal pain, but this was not significant. However, there may have been insufficient power to determine whether individual symptoms were associated with incomplete exams. Prior abdominal surgery $(47.4 \%$ vs $30.4 \% ; P=.005)$, hysterectomy $(46.3 \%$ vs $18.1 \% ; P=.001)$, and 
Table 2. Patient Characteristics According to Depth of Insertion

\begin{tabular}{lccc}
\hline & \multicolumn{2}{c}{ Insertion } & \\
\cline { 2 - 3 } & Complete & Incomplete & \\
Characteristics & $\mathrm{N}=427$ & $\mathrm{~N}=80$ & $P$ value \\
\hline Women, \% & 47.5 & 80.0 & $<.001$ \\
Age, mean years (SD) & $59.5(9.6)$ & $64.1(9.5)$ & $<.001$ \\
Weight, mean pounds & $188.8(45.0)$ & $176.0(37.9)$ & .021 \\
$\quad$ & & & \\
SyD) & & & \\
Abtoms, \% & 3.5 & 5.0 & $\mathrm{NS}$ \\
Constipation & 18.3 & 25.0 & $\mathrm{NS}$ \\
Diarrhea & 7.7 & 12.5 & $\mathrm{NS}$ \\
Pain & 13.6 & 20.0 & $\mathrm{NS}$ \\
Rectal bleeding & 18.3 & 21.3 & $\mathrm{NS}$ \\
Weight loss & 2.6 & 6.3 & $\mathrm{NS}$ \\
History of, \% & & & \\
Abdominal surgery & 30.4 & 47.4 & .005 \\
Hysterectomy & 18.1 & 46.3 & .001 \\
Reason, screening & 95.3 & 96.3 & $\mathrm{NS}$ \\
Previous sigmoidoscopy & 10.8 & 20.0 & .034 \\
Previous colonoscopy & 3.5 & 2.5 & $\mathrm{NS}$ \\
Preparation, inadequate, \% & 12.6 & 27.8 & .001 \\
\hline
\end{tabular}

* $P$ value for proportions by Pearson $\chi^{2}$, for means Student $t$ test.

${ }^{\dagger} \mathrm{NS}$, not significant $(P \geq .05)$.

inadequate bowel preparation $(27.8 \%$ vs $12.6 \%$; $P=.001)$ were significantly associated with incomplete examination.

Table 3 shows the univariate relative odds of incomplete depth of flex sig insertion according to patient characteristics. Patients with an incomplete examination were more likely to be women (OR $4.41 ; 95 \%$ CI, 2.47 to 7.88 ). Analysis of age distribution by sex and depth of flex sig insertion suggested that differences occurred only in older age groups. Women older than 70 years were more likely to have an incomplete examination (OR 2.27; $95 \%$ CI, 1.22 to 4.25$)$, as were men older than 75 years (OR 6.46; 95\% CI, 1.79 to 23.30). There was also a difference between men and women in the relative odds of incomplete insertion by weight loss (for women, OR 7.15; 95\% CI, 1.66 to 30.92; for men, OR 1.61; $95 \%$ CI, 0.29 to 8.97). For all other symptoms, the relative odds were not significant. Sex was an effect modifier for history of abdominal surgery (for women, OR 1.17; 95\% CI, 0.67 to 2.05; for men, OR 3.54; 95\% CI, 1.16 to 10.77). Sex may also have been an effect modifier for inadequate bowel preparation (for women, OR 3.58; 95\% CI, 1.75 to 7.31 ; for men, OR 2.03; $95 \%$ CI, 0.61 to 6.76 ). Age was an effect modifier for hysterectomy (for women $<70$ years, OR 4.54; $95 \%$ CI, 2.19 to 9.44 ; for women $>70$ years, OR 0.42 ;
Table 3. Patient Characteristics Associated with Incomplete Depth of Insertion

\begin{tabular}{lcc}
\hline Patient Characteristics & Odds Ratio & $95 \%$ CI \\
\hline Women versus men & 4.41 & $2.47-7.88$ \\
Age Categories & & \\
$\quad$ Women ( $\geq 70$ years vs $<70$ years) & 2.27 & $1.22-4.25$ \\
$\quad$ Men ( $\geq 75$ years vs $<75$ years) & 6.46 & $1.79-23.30$ \\
Symptoms & & \\
$\quad$ Abdominal distention & 1.45 & $0.47-4.47$ \\
$\quad$ Constipation & 1.49 & $0.85-2.62$ \\
$\quad$ Diarrhea & 1.71 & $0.80-3.62$ \\
Pain & 1.59 & $0.86-2.94$ \\
$\quad$ Rectal bleeding & 1.21 & $0.67-2.18$ \\
$\quad$ Weight loss & & \\
$\quad$ Women & 7.15 & $1.66-30.92$ \\
$\quad$ Men & 1.61 & $0.29-8.97$ \\
History of & & \\
$\quad$ Abdominal surgery & & \\
$\quad$ Women & 1.17 & $0.67-2.05$ \\
$\quad$ Men & 3.54 & $1.16-10.77$ \\
$\quad$ Hysterectomy & & \\
$\quad<70$ years & 4.54 & $2.19-9.44$ \\
$\quad \geq 70$ years & 0.42 & $0.14-1.23$ \\
$\quad$ Sigmoidoscopy & & \\
$\quad$ No previous versus previous & 2.01 & $1.11-3.88$ \\
Preparation, inadequate versus & & \\
$\quad$ adequate & & \\
$\quad$ Women & 3.58 & $1.75-7.31$ \\
Men & 2.03 & $0.61-6.76$ \\
\hline$\quad$
\end{tabular}

CI, confidence interval.

95\% CI, 0.14 to 1.23 ). According to univariate analysis, patients with no previous sigmoidoscopy were more likely to have an incomplete examination (OR 2.01; 95\% CI, 1.11 to 3.88).

Multivariate logistic regression models of incomplete depth of insertion were developed for both women and men. Separate models were developed because sex was an effect modifier for several factors, and multiple third-level interactions made interpretations of the relative odds values more difficult. For both men and women, history of previous flex sig and all symptoms other than weight loss were eliminated from the best model. For women, incomplete depth of insertion was related to inadequate preparation (OR 3.59; 95\% CI, 1.66 to 7.78). Age remained an effect modifier for hysterectomy, but large confidence intervals (because of low numbers) makes it difficult to interpret the magnitude of effect. Comparisons were made with the lowest risk group-women younger than 70 years with no hysterectomy. For women younger than 70 years, those with a hysterectomy were more likely to have an incomplete examination (OR 6.89; 95\% CI, 2.68 to 17.73). For women 70 years and older, the odds ratio for women with a hysterectomy (OR 2.68; 95\% CI, 0.96 to 7.46 
were similar to women without a hysterectomy (OR 4.79; 95\% CI, 2.27 to 10.12). There is significant overlap of the confidence intervals of the relative odds estimates for all these groups.

For men, age older than 75 years was related to incomplete depth of insertion (OR 6.51; 95\% CI, 1.72 to 30.40). In contrast to women, incomplete depth of insertion was related not to inadequate preparation but to abdominal surgery (OR 3.15; 95\% CI, 0.95 to 10.41 ) and weight loss (OR 9.62; $95 \%$ CI, 0.98 to 46.67$)$.

\section{Discussion}

The appeal of flexible sigmoidoscopy as a screening procedure lies in its wide availability, safety, relative ease of skill acquisition by most physicians, and ability to be offered on site with minimal ancillary support. Studies have shown that the majority of colon cancers and polyps are concentrated in the left colon, within the reach of the instrument. However, the capacity to detect polyps is dependent both on the depth and the proximal extent of insertion. Routine insertion to below the splenic flexure could result in an unacceptable degree of missed pathology (as detailed above). Recent studies have also demonstrated a more proximal distribution of colorectal cancers and polyps ${ }^{14}$ thereby increasing the need for a more complete examination.

Analysis of our series of 511 patients showed an association between incomplete depth of insertion (less than $50 \mathrm{~cm}$ ) and increasing age, female sex, hysterectomy, abdominal surgery (in men), weight loss (in men), and poor bowel preparation (in women). Holman et $\mathrm{al}^{9}$ also found a decreased depth of insertion in women with a history of pelvic surgery. Their analysis did not show any association with level of training, age, sex, history of abdominal surgery or symptoms at presentation. A retrospective study by Brill and Baumgardner, ${ }^{15}$ which analyzed the factors affecting the depth of insertion of the sigmoidoscope on 223 asymptomatic and symptomatic patients performed by residents at various levels of training, did show a correlation of depth of insertion with female gender, prior abdominal surgery and quality of the preparation. In their prospective study on 206 asymptomatic volunteers, Stewart et $\mathrm{al}^{6}$ found incomplete depth of insertion in up to a third of the patients and correlated this with female sex, previous ab- dominal surgery in women, high expectation of pain in women, and poor bowel preparation.

Although the previous studies have shown an increased risk of incomplete examination in women, we found that for women younger than 70 years, hysterectomy was a factor influencing incomplete examination. However, for women older than 70 years, the risk was independent of hysterectomy. Brill and Baumgardner ${ }^{15}$ found that previous abdominal surgery increased the likelihood of incomplete exams in both men and women, whereas our study found that abdominal surgery predicted unsatisfactory sigmoidoscopy only in men. Weight loss and age over 75 years predicted unsatisfactory sigmoidoscopy in men. We could not separate the risk of abdominal surgery from hysterectomy in women.

Using data collected over time from one endoscopist has certain limitations. Observer bias is possible because the endoscopist cannot be blinded to the existing variables affecting the depth of insertion. It is unlikely that any study would be designed in which the endoscopist has absolutely no knowledge of his subject. Does anticipation of problems during endoscopy based on the intake history or prior experience makes the practitioner unduly cautious and more determined to perform a complete examination? Experience improves our ability to obtain patient cooperation and enables us to continue with attempts at insertion, causing minimal damage.

Based on our logistic regression model, we estimate that the probability of an incomplete examination in women with poor bowel preparation was $25 \%$ (95\% CI, $20 \%$ to $32 \%$ ). In women under 70 years of age with a hysterectomy, the probability of an incomplete examination was about $40 \%$ (95\% CI, $20 \%$ to $63 \%$ ). If the woman also had a poor bowel preparation, the risk of incomplete screening was about $70 \%$ ( $95 \%$ CI, $51 \%$ to $84 \%$ ). We believe that with further study, it may be possible to develop a prediction model that could be used to determine whether a patient will have incomplete insertion during flex sig without sedation or analgesia (as it is now practiced). In those patients at high risk for incomplete insertion based on this model, clinicians could be ready to perform or repeat the procedure under sedation and/or analgesia or offer primary colonoscopic evaluation, based on patient preference. 
The high-risk characteristics associated with incomplete/difficult sigmoidoscopy and colonoscopy are similar. In colonoscopy, completion rates were found to be lower in the very young $(<20$ years) and the very old ( $>80$ years). The presence of certain symptoms (altered bowel habits, abdominal pain, diarrhea, constipation, hemorrhage), inflammatory bowel disease, and cancer) were also associated with incomplete examination ${ }^{16}$ Completion rates were also lower in women and after hysterectomy. ${ }^{17}$ However, even with these limiting factors, the colonoscopic completion rates are still approximately $90 \%$ in these subsets of patients. There is no evidence to suggest that incomplete colonoscopy is not associated with a higher complication rate. This information should be included in counseling patients about colorectal cancer screening options.

Audio and visual stimulation significantly reduces patient discomfort during screening flex sig. In a randomized trial, this was found to be an effective way of improving patient tolerance to routine screening flexible sigmoidoscopy and to improve patient compliance. ${ }^{18}$ Self-administered nitrous oxide also reduced patient discomfort during flexible sigmoidoscopy. ${ }^{19}$ This agent and distraction techniques have the potential to improve the success rate of flex sig, particularly if offered to patients who are more likely to have difficult examinations. Ketorolac administered 30 to $60 \mathrm{~min}$ utes before the procedure without sedation has been successfully used in colonoscopy with a $96 \%$ completion rate and also can be potentially offered to patients in whom difficult sigmoidoscopic examination is anticipated. ${ }^{20}$ Oral midazolam administered at a dose of $7.5 \mathrm{mg} 20$ minutes before sigmoidoscopy reduced anxiety and pain significantly during the procedure and may result in improved depths of insertion. ${ }^{21}$

\section{Conclusion}

Until colonoscopy is freely available or we have a noninvasive test capable of matching its sensitivity in screening for colorectal cancer, flex sig remains an important screening modality that can be offered for average risk patients over the age of 50. As it now stands, flexible sigmoidoscopy remains an easily administered screening procedure that can be offered to the population at risk by both physicians and physician extenders, with minimal financial and organizational outlay.
The crux is in examining as much of the colon as feasible, thereby minimizing missed pathology. Herein lies the importance of patient selection. Further study is necessary to determine whether a predictive model can be developed that would be useful in selecting patients most appropriate for this examination. In patients in whom successful sigmoidoscopy is unlikely, consideration should be given to performing sigmoidoscopy under sedation, analgesia, distraction techniques, or offering primary colonoscopy.

\section{References}

1. Peipins LA, Sandler RS. Epidemiology of colorectal adenomas. Epidemiol Rev 1994;16:273-97.

2. Tedesco FJ, Waye JD, Avella JR, Villalobos MM. Diagnostic implications of the spatial distribution of colonic mass lesions (polyps and cancers). A prospective colonoscopic study. Gastrointest Endosc 1980; 26:95-7.

3. Johnson DA, Gurney MS, Volpe RJ, et al. A prospective study of the prevalence of colonic neoplasms in asymptomatic patients with an age- related risk. Am J Gastroenterol 1990;85:969-74.

4. Lieberman DA, Smith FW. Screening for colon malignancy with colonoscopy. Am J Gastroenterol 1991;86:946-51.

5. Olynyk JK, Aquilia S, Fletcher DR, Dickinson JA. Flexible sigmoidoscopy screening for colorectal cancer in average-risk subjects: a community-based pilot project. Med J Aust 1996;165:74-6.

6. Stewart BT, Keck JO, Duncan AV, Santamaria NM, Allen P. Difficult or incomplete flexible sigmoidoscopy: implications for a screening programme. Aust N Z J Surg 1999;69:19-21.

7. PainterJ, Saunders DB, Bell GD, Williams CB, Pitt $\mathrm{R}$, Bladen J. Depth of insertion at flexible sigmoidoscopy: implications for colorectal cancer screening and instrument design. Endoscopy 1999;31:227-31.

8. Lehman GA, Buchner DM, Lappas JC. Anatomical extent of fiberoptic sigmoidoscopy. Gastroenterology 1983;84:803-8.

9. Holman JR, Marshall RC, Jordan B. Technical competency in flexible sigmoidoscopy. J Am Board Fam Pract 2001;14:424-9.

10. Gillespie PE, Chambers TJ, Chan KW, Doronzo F, Morson BC, Williams CB. Colonic adenomas-a colonoscopy survey. Gut 1979;20:240-5.

11. Shinya H, Wolff WI. Morphology, anatomic distribution and cancer potential of colonic polyps. An analysis of 7000 polyps endoscopically removed. Ann Surg 1979;190:679-83.

12. Pierzchajlo RPJ, Ackermann RJ, Vogel RL. Colonoscopy performed by a family physician. A 
case series of 751 procedures. J Fam Pract 1997;44: 473-80.

13. Lieberman DA, Weiss DG, Bond JH, Ahnen DJ, Garewal H, Chejfec G. Use of colonoscopy to screen asymptomatic adults for colorectal cancer. Veterans Affairs Cooperative Study Group 380. N Engl J Med. 2000;343:162-8.

14. McCallion K, Mitchell RM, Wilson RH, et al. Flexible sigmoidoscopy and the changing distribution of colorectal cancer: implications for screening. Gut 2001;48:522-5.

15. Brill JR, Baumgardner DJ. Establishing proficiency in flexible sigmoidoscopy in a family practice residency program. Fam Med 1997;29:580-3.

16. Church JM. Complete colonoscopy: how often? And if not, why not?. Am J Gastroenterol 1994;89:556-60.
17. Cirocco WC, Rusin LC. Factors that predict incomplete colonoscopy. Dis Colon Rectum 1995;38: 964-8.

18. Lembo T, Fitzgerald L, Matin K, Woo K, Mayer EA, Naliboff BD. Audio and visual stimulation reduces patient discomfort during screening flexible sigmoidoscopy. Am J Gastroenterol 1998;93:1113-6.

19. Harding TA, Gibson JA. The use of inhaled nitrous oxide for flexible sigmoidoscopy: a placebo-controlled trial. Endoscopy 2000;32:457-60.

20. Hopper W, Kyker KA, Rodney WM. Colonoscopy by a family physician: a 9-year experience of 1048 procedures. J Fam Pract 1996;43:561-6.

21. Kuganeswaran E, Clarkston WK, Cuddy PG, et al. A double-blind placebo controlled trial of oral midazolam as premedication before flexible sigmoidoscopy. Am J Gastroenterol 1999;94:3215-9. 